\title{
Percepción de seguridad en espacios públicos de conjuntos habitacionales de interés social: Poza Rica y
} Coatzintla, 2000 a 2016

Perceptions of security in public spaces of social housing complexes: Poza Rica and Coatzintla, 2000 - 2016

\section{Selenne Galeana Cruz *}

Recibido: 14 de agosto de 2017

Aceptado: 12 de marzo de 2018

\section{Resumen}

El artículo ofrece un análisis de la percepción de seguridad en los usuarios del espacio público en conjuntos habitacionales, con el objetivo de esclarecer su incidencia en la apropiación y uso y, por tanto, en la satisfacción. Para ello, se toman en cuenta las dimensiones morfológica y urbana, arquitectónica, y social. Se ocupa el método cualitativo (observación y entrevistas), y el cuantitativo (encuesta). El estudio se realiza en dos conjuntos habitacionales de interés social en la zona metropolitana de Poza Rica y Coatzintla, Veracruz, México. En el primer caso es un condominio llamado Geovillas del Real y, en el segundo, un fraccionamiento cuyo nombre es Los Mangos 1 y 2 . Se concluye que la percepción de seguridad en los habitantes de estos conjuntos está debilitada debido al miedo a ser violentados por la delincuencia y el crimen organizado. Por otro lado, las condiciones precarias de vivienda y del espacio público, así como la carencia de servicios básicos han sido factores determinantes en la construcción del concepto y noción de inseguridad más que de seguridad, ya que restringen y transforman las formas de apropiación y uso, y, por ende, impiden la obtención de satisfacción.

Palabras clave: apropiación y uso del espacio público, espacio público, percepción de seguridad, satisfacción con el entorno.

\begin{abstract}
This paper analyzes the citizen's perception of security in public spaces, the objective is to determine the incidence of the appropriation of public space, and as a result, its satisfaction on the context involved. The morphologic and urban, as well as the architecture and social dimensions, are taken into consideration in this research. Qualitative (direct observation and interviews) and quantitative (survey) methods are used. The study took place in two public housing complexes in the metropolitan area of Poza Rica and Coatzintla Veracruz Mexico. The first case of study is a condominium called Geovillas del Real, the second case is a neighborhood called Los Mangos first and second stage. We have concluded that the habitant's perceptions of security are weak, they are afraid of the violence produced by delinquency and organized crime. On the other hand, the precarious conditions of public space, as well as the lack of municipal utilities have been factors that hold back the construction of the concept of security, due to this situation there is a restriction and transformation about the appropriation of the usage of public space, the satisfaction is not reached by the citizens.
\end{abstract}

Keywords: appropriation of the public space, comfort, perceptions of security, public space.

\footnotetext{
* Facultad de Arquitectura de la Universidad Nacional Autónoma de México, Ciudad de México, México. Contacto: arquisel@hotmail.com
}

Cómo citar: Galeana, S. (2018). Percepción de seguridad en espacios públicos de conjuntos habitacionales de interés social: Poza Rica y Coatzintla, 2000 a 2016. Revista de Urbanismo, 38, 1-14. http://dx.doi.org/10.5354/0717-5051.2018.47084 


\section{Introducción}

En este artículo se estudia la percepción de seguridad en los usuarios del espacio público en dos conjuntos habitacionales de interés social de la zona metropolitana de Poza Rica y Coatzintla, Veracruz: Geovillas del Real y Los Mangos 1 y 2. El espacio público se entiende como un ámbito de uso colectivo, escenario de encuentros casuales y previstos que reproduce la interacción cotidiana de un grupo, llevándolo a escala barrial. El espacio público abarca jardines, andadores, pasillos, vestíbulos, banquetas, vías, áreas de convivencia y escalones.

Esta definición está relacionada con la dimensión perceptual, social y funcional del entorno construido y particularmente con la sensación de seguridad (Carmona, Health, Oc, \& Tiesdell, 2003). En este estudio se observa que el espacio público está asociado con peligro, miedo y desconfianza, es decir, con la noción de seguridad en sentido negativo, lo que lleva a concluir que los habitantes lo asocian con inseguridad más que con seguridad.

Los conjuntos habitacionales objeto de estudio representan un tipo de construcción cuya planeación deriva de la política habitacional mercantil, la cual otorga mayor importancia al beneficio económico y financiero de las empresas productoras (Schteingart y Patiño, 2006). Lo anterior tuvo su despunte a partir de 1992 y 1993 cuando se hicieron transformaciones a los marcos legislativo, programático e institucional de la política de vivienda y se creó el Programa Especial para el Fomento y Desregulación de la Vivienda (PFDV-93) a nivel nacional. Con este nuevo programa se implementaron algunas reformas en materia de vivienda y desarrollo urbano que se consolidaron en el Programa Especial para el Fomento y Desregulación de la Vivienda (PFDV-95), cuya línea de política se agudizó con el Programa Especial para el Fomento y Desregulación de la Vivienda (PFDV-01) (Schteingart y Patiño, 2006). La línea de política tiene la intención de industrializar y generar una economía de escala, la cual consiste en ahorrar tiempo y costos de construcción y disminuir la mano de obra.

En este estudio se analizan los conjuntos habitacionales Geovillas del Real y Los Mangos 1 y 2, debido a que el primero (construido en 2008-2010, con 2.028 viviendas) derivó del Programa Especial para el Fomento y Desregulación de la Vivienda (PFDV-2007), en tanto que el segundo (construido en 2000-2003, con 546 viviendas) se basó en el Programa Especial para el Fomento y Desregulación de la Vivienda (PFDV-95).

Los Mangos 1 y 2, la construcción menos reciente de este estudio, es el resultado de las reformas realizadas en la política habitacional a partir de 1992 y 1993, cuando se pasó de la construcción tradicional a la industrializada, todavía con la participación directa de empresas constructoras locales. Por su parte, Geovillas del Real, la construcción más reciente, es el tipo de conjunto urbano resultado de los cambios hechos al sistema de producción en 2001, de entre los que destacan la construcción en serie y la homologación de criterios de diseño y materiales que no toman en cuenta las características propias de cada lugar. A partir del inicio de este segundo milenio las grandes empresas inmobiliarias tomaron el papel protagónico.

Ambos cambios, tanto el de la transición de la producción tradicional a la industrial con la participación directa de las constructoras locales como el del industrial producido en serie, con la homologación de criterios de diseño y la participación de grandes empresas inmobiliarias, fueron resultado del desarrollo del sistema industrial en la producción de vivienda de interés social.

Como dicen Schteingart y Patiño (2006) se empezó a perder la importancia de la orientación social de la vivienda y su vinculación con el desarrollo urbano; el financiamiento, el diseño y la construcción quedó en manos del capital privado, lo cual tuvo implicaciones en la habitabilidad.

El desarrollo que tuvieron los municipios de Poza Rica y Coatzintla fue producto del descubrimiento e industrialización del petróleo a principios del siglo XX. Dicho recurso natural trajo el crecimiento económico en México a partir de la expropiación petrolera en 1938.

Según el Programa Municipal de Desarrollo Urbano de Poza Rica (2009), este municipio fue punto clave en la logística administrativa y económica del país. De hecho, su consolidación en la actividad petrolera atrajo a personas de los estados de Puebla, Guadalajara, Distrito Federal e Hidalgo, de otros países como Estados Unidos e Inglaterra, y de pueblos aledaños como Papantla, Tuxpan y Coyutla, entre otros. En particular, tuvo influencia en la localidad más cercana: Coatzintla. Así fue como Poza Rica se expandió hasta formar la zona metropolitana.

Los empleos formales derivados de la industria petrolera atrajeron a otras industrias y empresas y así, hubo un aumento en la derrama de los salarios y de la población. Al mismo tiempo, creció la necesidad de vivienda tanto por el aumento natural de los lugareños, como por la migración que atraía el mercado petrolero.

Esta demanda de vivienda también se incrementó por parte de los trabajadores asalariados de bajo ingreso, que laboraban tanto en el rubro del petróleo como fuera de este, en instancias públicas y/o privadas. Por lo anterior, la zona se vio beneficiada desde 1978 con el Programa Nacional de Vivienda de ese año, que dio lugar a la construcción de los primeros conjuntos habitacionales de interés social, los cuales constituyen el 7,45\% del total actual de vivienda de interés social (VIS) de la zona 
metropolitana ${ }^{1}$. En total los conjuntos de ese año suman 1.400 viviendas.

Para la década de los ochenta, la proporción fue de 13,25\% más de construcción, con 2.488 VIS; en los noventa se construyó un $31,50 \%$ más, con 5.915 VIS. A partir del año 2000 se produjo la mayor producción de VIS, aumentando al $47,80 \%$ de la construcción actual, equivalente a 8.985 viviendas.

Más tarde, en 2008, a la par de la Reforma energética, se puso en marcha el proyecto Activo Integral Aceite Terciario del Golfo (ATG). La zona metropolitana de Poza Rica y Coatzintla se convirtió en un lugar de operación y logística, lo cual atrajo a diferentes desarrolladores inmobiliarios. Así se inició la construcción del primer conjunto urbano masivo Geovillas del Real, donde en mayor medida los adquirientes de vivienda fueron los obreros de empresas subcontratadas por Petróleos Mexicanos (Pemex) y empleados de pequeñas empresas o establecimientos comerciales locales, dependientes de la inyección económica petrolera².

Este auge económico surgido en 2008 solo duró cinco años, de tal forma que la zona metropolitana de Poza RicaCoatzintla se vio afectada de manera inmediata con la pérdida de fuentes laborales, aproximadamente $130 \mathrm{mil}$ suspensiones ${ }^{3}$ entre trabajadores extranjeros, nacionales y locales. Se acabó la buena inyección económica que duró de 2008 a principios de 2013 y los desempleados se quedaron con deudas, entre ellas, la de la vivienda.

A la crisis laboral se sumó, desde principios de 2013, la crisis financiera a nivel nacional de los desarrolladores inmobiliarios (González, 2014), entre ellos la empresa que construyó Geovillas del Real. Tal circunstancia incidió en las condiciones físicas del conjunto habitacional, así como en la falta de mantenimiento, equipamiento y servicios básicos.

En particular, el abastecimiento de energía eléctrica comenzó a ser intermitente, ya que disminuyó la cantidad de diésel para el funcionamiento de las plantas de luz; decayó el mantenimiento del espacio público a consecuencia de la indiferencia al cuidado y al pago de cuotas por parte de los habitantes, lo que ocasionó la ausencia de vigilancia privada 4 . Además, tanto en Geovillas del Real como Los Mangos 1 y 2 empezaron los robos a viviendas habitadas, los asaltos a transeúntes, el aumento de viviendas habitadas por parte de personas

\footnotetext{
${ }^{1}$ El cien por ciento corresponde a 18.788 VIS en la zona metropolitana de Poza Rica-Coatzintla, Veracruz.

2 Esta información se obtuvo en una entrevista con un administrativo de la empresa Geo, en julio de 2013.

3 Información declarada por Amador Leonardo, diputado federal en Veracruz. Asimismo, por testimonio de María del Pilar Rosario Moreno, representante de la Asociación Nacional de Hoteles y Moteles. Ver Punto de Partida. 25 de septiembre de 2015. "Cruda realidad: Poza Rica sin empleos, sin inversión y sin desarrollo", Denise Maerker, conductora.
}

externas al conjunto habitacional y, según lo expresado en las entrevistas que se hicieron a los habitantes de Geovillas del Real en agosto de 2015, este se convirtió en guarida del crimen organizado. Complementariamente, en Los Mangos 1 y 2, en 2010, un niño fue secuestrado por un grupo paramilitar criminal y el miedo incrementó notablemente a raíz de ese suceso.

En ambos conjuntos la crisis económica petrolera e inmobiliaria conllevó a la falta de empleo y a la precarización de las condiciones físicas. En el contexto social nacional, la falta de seguridad se incrementó a partir del 2006 cuando se inició la lucha armada contra el crimen organizado (Nava, 2012). Así, el panorama de seguridad del Estado de Veracruz se ha visto quebrantado paulatinamente en los últimos años, y las ciudades de Poza Rica y Coatzintla no han quedado exentas de esa problemática.

Cuando aquí se refiere al "crimen organizado" se habla de una organización criminal y paramilitar mexicana denominada "los Zetas", cuyas principales acciones delictivas son el tráfico de drogas, la extorsión, el secuestro, el homicidio, el tráfico de personas y el robo a mano armada. Estos hechos suceden en el espacio público tanto fuera como dentro de los conjuntos habitacionales. Al producirse en las calles, los parques, las plazas, o cualquier lugar público abierto, la población percibe estos espacios como inseguros.

En un primer acercamiento a la población de los conjuntos habitacionales bajo estudio, se percibió vulnerabilidad, por ello, para realizar la presente investigación se plantearon las siguientes preguntas:

- ¿Cómo perciben los habitantes el espacio público?

- ¿A partir de dónde y cómo la gente percibe la seguridad o la carencia de ella: inseguridad (peligro, miedo y desconfianza)?

- ¿Cómo afecta la percepción de seguridad-inseguridad en la apropiación y uso cotidiano del espacio público?

El objetivo principal de esta investigación fue analizar los factores y prácticas que conllevaban a la percepción de inseguridad de la población y cuánto de ello podía incidir en la construcción de la seguridad a través de la satisfacción obtenida del espacio público. Para lograr lo

\footnotetext{
${ }^{4}$ En la actualidad, los propios habitantes gestionan y pagan los servicios de mantenimiento, recolección de basura y vigilancia. La energía eléctrica es privada y pública según el área correspondiente; la Comisión Federal de Electricidad (CFE) abastece a $50 \%$ de las viviendas del conjunto habitacional; por otro lado, $50 \%$ no cuenta con este servicio, puesto que se abastecen por medio de un transformador que trabaja con diésel, a cargo de la empresa inmobiliaria. Esta información fue recolectada en campo.
} 
anterior se analizaron las dimensiones morfológica y urbana, arquitectónica, y social, en relación con los servicios básicos (iluminación, mantenimiento y vigilancia).

La hipótesis fue que la presencia del crimen organizado, los secuestros, los asesinatos, los robos y los asaltos en un contexto de crisis económica y social, son prácticas que aumentan la incertidumbre de los usuarios del espacio público, aun cuando este se ubique dentro de un conjunto habitacional. Lo anterior se intensifica con el diseño urbano y arquitectónico amurallado, el cual impide el intercambio físico y visual entre espacio público y privado. Las características del terreno, la cercanía o existencia de referentes de peligro como los canales de agua pluvial o fluvial, las condiciones precarias de los materiales, el deterioro prematuro y la carencia de servicios básicos como mantenimiento, vigilancia e iluminación, son factores que inciden en la percepción de una seguridad inexistente y derivan en la solidificación de la sensación humana de inseguridad. Esto ocasiona una exigua apropiación y uso cotidiano del espacio público.

\section{Aproximaciones teóricas}

Lo público refiere a un espacio abierto que es asequible para todos los miembros de una comunidad; remite a los bienes y servicios públicos, a lo que es de interés o de utilidad común, a lo que atañe al colectivo cuyas reglas y normas de uso deben ser visibles y conocidas por todos (Rabotnikof, 2011). Uno de los desafíos del espacio público consiste en vivirse "como espacio de la expresión colectiva, la vida comunitaria, del encuentro, del intercambio cotidiano, de las afirmaciones y confrontaciones ciudadanas" (Jiménez y Garnica, 2016, p. 75), es un lugar de convivencia e interacción social (Cruz Muñoz e Isunza, 2017).

El espacio público, para fines de esta investigación, se define como aquel ámbito común construido tanto por elementos urbanos y arquitectónicos como por relaciones de encuentro, mismo que se modifica y construye a partir de la apropiación y el uso cotidiano de un grupo a escala barrial; el espacio público también es escenario de relaciones de cooperación, enfrentamiento e indiferencia, y su accesibilidad remite a la localización, la cual se analiza a través de la permeabilidad (física y visual), donde la gente puede o no caminar o mirar, como proponen Bentley, Alcock, Murrain, MacGlynn y Smith (1999), en relación con las condiciones (Urbano Paz y Delpino Chamy, 2015). La accesibilidad física y visual es un indicador de la interacción social y tiene que ver con "añadir más ojos a los que normalmente miran a la calle", se trata también de "inducir a los que viven en las casas a observar la calle en número y ocasiones suficientes" Jacobs $(1973$, p. 6), es decir, la permeabilidad entre el espacio público y el privado es un mecanismo que acrecienta la percepción de seguridad y, con ello, la sensación de satisfacción aumenta.

Por su parte, abordar el concepto de satisfacción en el espacio público implica tener en cuenta la noción de calidad de vida, que según la Organización Mundial de la Salud (World, Health Organization, WHO, 2018) se define como:

... la percepción que un individuo tiene de su lugar en la existencia, en el contexto de cultura y del sistema de valores en los que vive y en relación con sus expectativas, sus normas y sus inquietudes. Se trata de un concepto amplio que está influido de un modo complejo por la salud física del sujeto, su estado psicológico, su nivel de independencia, sus relaciones sociales, así como su relación con los elementos esenciales de su entorno. (WHO, 2018)

En el contexto institucional mexicano, el Programa Nacional de Vivienda 2014-2018 define la calidad de vida como "tener buenas condiciones de vida objetivas y un alto grado de bienestar subjetivo; incluye también la satisfacción colectiva de necesidades ... en adición a la satisfacción individual de necesidades" (2014, p. 29).

Otra definición para calidad de vida corresponde al "bienestar como satisfacción de preferencias y necesidades, obtención de un estado del mundo que depende de las capacidades de las personas para elegir el estilo de vida" (Nussbaum y Sen, 1996, p. 18). Ahora bien, según Alguacil (2000), la calidad de vida está relacionada con el grado óptimo de satisfacción de las necesidades humanas individuales y/o colectivas. Por lo anterior, en este estudio se remite al concepto de satisfacción como las condiciones de vida idóneas o pésimas que conducen a estar bien o mal, según la valoración (Mejía, 2012), en relación con las necesidades básicas individuales como son dormir, descansar, comer, tener higiene y las necesidades colectivas como la interacción y relación con el otro. Esta definición alude a la disconformidad o a la adaptación que hay entre los intereses y los atributos que tiene el espacio público - si se lleva al plano que compete- como satisfactor; es decir, como cuerpo que solventa las necesidades colectivas y las expectativas de las personas, Levi y Anderson (1980), citado por Rueda (1997, p. 2).

La satisfacción, como indicador de calidad de vida, es evaluada entonces a partir del vínculo real/objetivo entre vivienda y espacio público, y la percepción/subjetiva que los usuarios tengan de ella. Como indicadores objetivos se destacan las características físicas, y como términos subjetivos las prácticas y las relaciones que conllevan a la apropiación y al uso del espacio público (Durán, Esquivel y 
Giglia, 2000) las cuales (prácticas y relaciones) responden a la percepción que se tiene de este.

El proceso de percibir el espacio, el natural y el construido, significa conocer el ambiente inmediato por medio de los sentidos. Tal proceso implica reinterpretar imágenes del medio físico momentos después de captar el entorno, hecho que incita a diversos tipos de actitud hacia él (Holahan, 2011). Asimismo, Carmona et al., (2003) expresan que lo perceptual está ligado al sentido de lugar y es inherente a lo social - que puede ser entendido por la noción de seguridad-, y la funcional, debido al diseño del entorno construido. En este sentido, la percepción de seguridad está paradójicamente relacionada con el peligro, el miedo y la desconfianza, es decir, con la inseguridad. La percepción de inseguridad es una de las piezas clave para entender la apropiación y el uso del espacio público, además de ser un indicador muy importante de la calidad de vida de las personas (Fraile, 2007).

De acuerdo con Jasso (2013, p. 16) la percepción de inseguridad se puede entender "como tal - una percepción-, en cuanto a que la persona se sitúa frente a las circunstancias y emite un juicio sobre las posibilidades de ser víctima de un delito, basándose en sus conocimientos y sus actividades cotidianas". Dichas circunstancias están relacionadas con la incivilidad, aunque puede derivar de factores relacionados con el lugar o medio ambiente que ponen en riesgo su integridad física, lo cual se compagina con el contexto social y el modo de habitar.

En coincidencia con Giglia (2012), el proceso de habitar consiste en las prácticas y las representaciones que un sujeto individual o colectivo lleva a cabo. Esta apropiación implica la trasformación física y social del espacio público mediante prácticas cotidianas. Por ello, el habitar supone la apropiación y el uso de la realidad percibida, como plantea Maycotte (2010). Esto alude al espacio practicado que se puede entender como "esa relación recíproca entre el lugar y la sociedad" (García y Peralta, 2016, p. 80).

Existen escasos estudios que hayan profundizado acerca de la percepción de seguridad en conjuntos habitacionales de interés social y los que se han realizado se han orientado a procesos de exclusión y segregación social, pobreza, aislamiento y vulnerabilidad social. Por tal motivo, el enfoque del análisis parte de la conjunción de varias investigaciones que han indagado en torno al tema de la vivienda de interés social con base en indicadores de calidad de vida y el espacio público, en conjuntos habitacionales para población de recursos limitados.

Del estudio realizado por Giglia (2000, p. 87-112) en Monteruscello, Italia, se retoma el método para analizar la construcción de hechos sociales, las relaciones sociales y la apropiación y el uso en la vida cotidiana, suscitados por el encuentro casual en los espacios de circulación, en los espacios entre edificios, en las escaleras y en los pasillos, entre otros.

Segovia (2005) estudia el contexto socioespacial en tres conjuntos habitacionales de interés social, localizados en la periferia sur de la ciudad de Santiago de Chile. La autora explica que las características espaciales se trasladan a lo social: la estrechez de los espacios públicos denota percepción de inseguridad del espacio público. En coincidencia con su enfoque, se parte del análisis de la accesibilidad con base en la localización; de los componentes arquitectónicos en torno a las características físicas y el diseño del espacio público (calidad del material, distribución, mantenimiento $y$ servicios).

Otro trabajo importante es el realizado por Durán et al. (2000) en conjuntos habitacionales del Distrito Federal de México. Parten del análisis del proceso de uso y las condiciones de vida, de manera que proponen tres dimensiones de análisis: física, económica y social, y urbana. De la dimensión física de adopta la observación del estado constructivo, así como la disponibilidad de servicios, ya señalado desde el trabajo de Segovia (2005). De la dimensión social se retoma la importancia del mantenimiento del espacio colectivo como indicador de organización vecinal; $\mathrm{y}$, por último, los problemas de inseguridad, delincuencia y vigilancia (Durán et al., 2000).

En cambio, Alguacil (2000) propone tres dimensiones de análisis: el estado físico, la accesibilidad y los servicios básicos, lo cual confluye con la propuesta de Durán et al. (2000) sobre la dimensión urbana, y con Segovia (2005) respecto de la localización de los conjuntos habitacionales y los servicios básicos (iluminación y vigilancia); la social; y la emocional.

Por otra parte, Rueda (1997) propone cuatro dimensiones de análisis: el bienestar, la calidad ambiental, el aspecto psicosocial -coincidente con la dimensión social de Durán et al., (2000) y Alguacil (2000)-, y el entorno sociopolítico.

En suma, las propuestas se centran en varias dimensiones de análisis, de modo que para fines de esta investigación se concretaron en las dimensiones

- morfológica y urbana (accesibilidad, mantenimiento y referentes de peligro);

- $\quad$ arquitectónica (diseño y calidad de construcción); y

- $\quad$ social (incivilidad, vigilancia y desconfianza), que de manera horizontal involucra los servicios básicos (iluminación, mantenimiento y vigilancia).

Es importante indicar la fuerte relación existente entre las dimensiones analizadas, pero para fines del estudio se exponen de manera diferenciada. 


\section{Metodología: enfoque metodológico y técnicas}

La investigación compaginó con las tres escalas de análisis morfológicas: la conexión entre ciudad y conjunto habitacional; entre la vivienda y su posición en el conjunto habitacional; y entre el espacio público y la vivienda.

Se trabajó una parte cualitativa que se refería a la observación directa y las entrevistas semiestructuradas de tipo incidental. Se hizo observación constante y se entrevistó a miembros del comité vecinal y algunos transeúntes, en octubre de 2013, en agosto de 2014 y en febrero de 2015. Esto es relevante porque en el proceso se registraron transformaciones en la organización del espacio, el suministro de servicios básicos y el contexto social, por lo tanto, en la manera de vivir y percibir seguridad.

El número de cuestionarios se fijó a partir de la repetición a las respuestas de las preguntas y se efectuaron 41 entrevistas. En Geovillas del Real se hicieron 26 entrevistas: en primer lugar, a tres miembros del comité vecinal (dos en 2013 y uno en 2015) y a 23 transeúntes (tres en 2013, cinco en 2014 y 15 en 2015). En Los Mangos 1 y 2 se hicieron 15 entrevistas: primeramente, a dos miembros del comité vecinal (uno en 2014 y otro en 2015) y a 13 transeúntes (dos en 2014 y once en 2015) (Tabla 1).

Otra parte del análisis se retroalimentó con una fase cualitativa, por medio de una encuesta que se llevó a cabo en febrero de 2015. Para la selección de la vivienda se ocupó el muestreo aleatorio simple y se utilizó la fórmula estadística $Z_{p}=(1,96)^{2}(0,5)(0,5) /(0,05)^{2}$ con un $95 \%$ de confianza y un error de precisión del 5\%; sin embargo, fueron solo 30 cuestionarios en cada conjunto habitacional los contemplados para la investigación, pues la presencia del crimen organizado y el desempleo generaron desconfianza entre los entrevistados (Tabla 1$)^{5}$.

Tabla 1

Enfoque metodológico y técnicas

\begin{tabular}{|c|c|c|c|c|c|c|c|}
\hline Metodología & \multicolumn{6}{|c|}{ Cualitativa } & \multirow{2}{*}{ Cuantitativa } \\
\hline Años & & 2013 & & & & 2015 & \\
\hline \multirow[t]{2}{*}{ Técnicas } & \multicolumn{6}{|c|}{ Observación } & \\
\hline & \multicolumn{6}{|c|}{ Entrevista } & Encuesta \\
\hline Muestra & $\begin{array}{l}\text { Comité } \\
\text { vecinal }\end{array}$ & Transeúnte & $\begin{array}{l}\text { Comité } \\
\text { vecinal }\end{array}$ & Transeúnte & $\begin{array}{l}\text { Comité } \\
\text { vecinal }\end{array}$ & Transeúnte & Vivienda \\
\hline Geovillas de Real & 2 & 3 & 0 & 5 & 1 & 15 & 30 \\
\hline $\begin{array}{c}\text { Mangos } \\
1 \text { y } 2\end{array}$ & 0 & 0 & 1 & 2 & 1 & 11 & 30 \\
\hline
\end{tabular}

Fuente: Elaboración propia.

\section{Resultados y discusión}

Para facilitar la evaluación de los conjuntos habitacionales estos se dividieron en zonas, y se tomaron en cuenta aquellas donde hubo mayor número de respuestas relacionadas con la percepción de inseguridad. En Geovillas del Real, las áreas donde hay mayor percepción de peligro, miedo y desconfianza corresponde a las zonas II (el paradero de camiones, el área de comercio informal y la plaza comercial principal), VI y VII, asimismo la vía colectora y el acceso secundario (Figura 1).

${ }^{5}$ Para referir a las personas que participaron en la entrevista o en la encuesta, se utilizó un sistema de referencia conformado con el número que le corresponde como entrevistado o encuestado, las iniciales del conjunto habitacional y el año en el que se llevaron a cabo. A saber:
Entrevistado 4, Geovillas del Real, 2015 es igual a ENT4-GR, 2015 o Encuestado 5, Geovillas del Real, 2015 es igual a ENC5-GR, 2015. Para Los Mangos 1 la clave es LM1 y para Los Mangos 2 es LM2. 


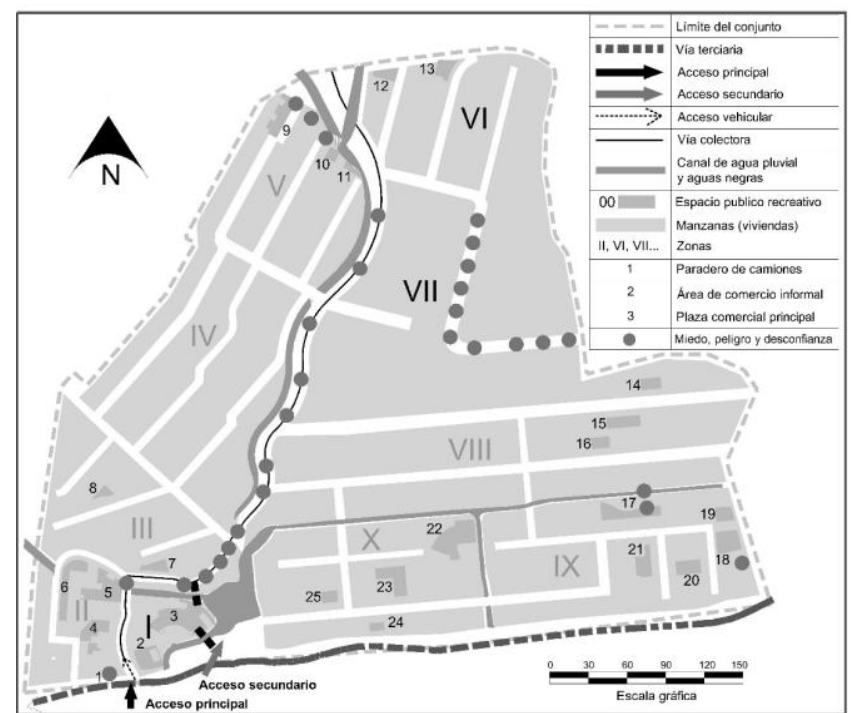

Figura 1. Geovillas del Real. Fuente: Elaboración propia.

En Los Mangos 1 y 2 se mencionaron el peligro, el miedo y la desconfianza, tanto en la zona de Los Mangos 1 (el acceso principal, un nicho religioso, una jardinera en banqueta, el acceso principal del espacio público recreativo A y una vía local), como en la zona de Los Mangos 2 (el canal de agua fluvial y el espacio C) (Figura 2).

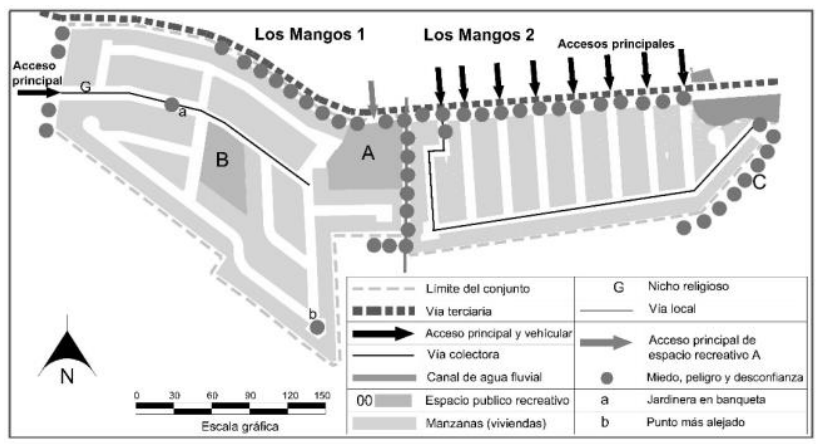

Figura 2. Los Mangos 1 y 2.

Fuente: Elaboración propia.

Las zonas de ambos conjuntos habitacionales se analizaron a partir de las dimensiones contempladas en el estudio y las respectivas variables que las sintetizan.

\section{Dimensión morfológica y urbana: accesibilidad, mantenimiento y referentes de peligro}

En Geovillas del Real, en el perdedero de camiones se percibe inseguridad, pues colinda con el borde cerrado del límite del conjunto y la barda tiene en la actualidad una altura de 2 metros (m). Además, su permeabilidad visual desde la plaza comercial principal y el área de comercio informal es restringida (Figura 3), a lo cual se suma la existencia de una zona con maleza y basura.

El acceso secundario es otro referente de inseguridad (Figura 3). En sus bordes colinda, en un lado, con el canal de agua pluvial y aguas negras y con terreno baldío; en el otro, con la barda perimetral del conjunto habitacional, lo que ocasiona que su permeabilidad visual sea limitada. Además, la maleza, la basura y las culebras están presentes cotidianamente.

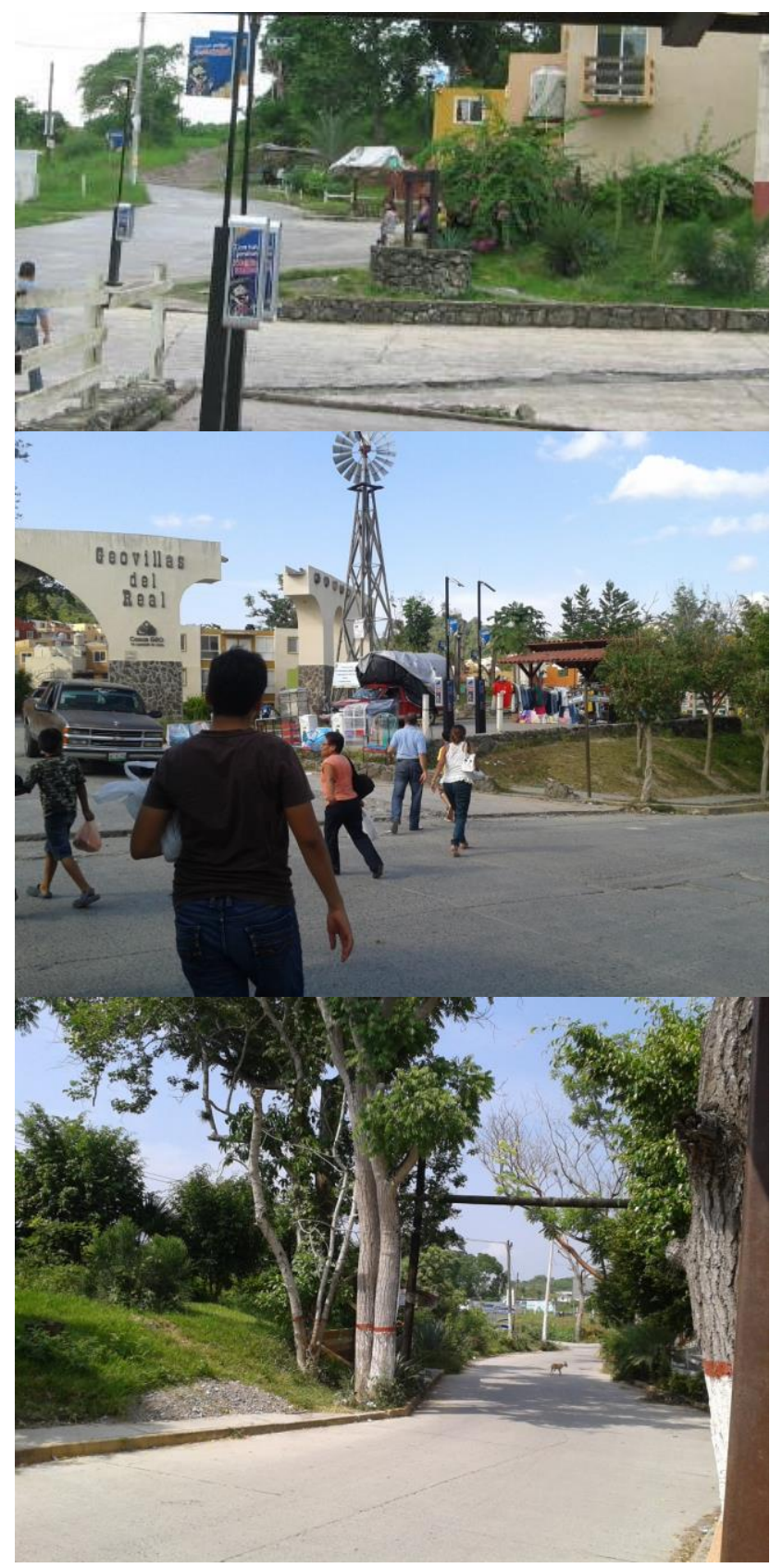

Figuras 3, 4 y 5. Paradero de camiones, área de comercio informal y plaza comercial principal, y acceso secundario.

Fuente: Elaboración propia. 
La percepción de inseguridad también se asocia a la vía colectora (Figuras 3, 4 y 5). El borde de ambos lados de dicha vía está conformado por las fachadas traseras y laterales de las viviendas, lo que reduce la permeabilidad visual entre el espacio público y privado. Jacobs (2011) expresa algo contrario a lo que sucede en Geovillas de Real:

... los edificios de una calle equipada para superar la prueba de los desconocidos $y$, al mismo tiempo, procurar seguridad a vecinos y desconocidos, han de estar orientados de cara a la calle. No deben dar su espalda ni sus muros a la calle dejándola así ciega ( $p$. 61).

Tal configuración aumenta la vulnerabilidad de los transeúntes de ser víctimas de la delincuencia, tal y como ha sucedido. Un residente comenta: "lo que es la glorieta... trataron de asaltar a una persona, han asaltado a muchos" (ENT2-GR, 2014). Dicha configuración urbana y arquitectónica remite a la incivilidad social que aprovecha la falta de permeabilidad para transgredir la seguridad.
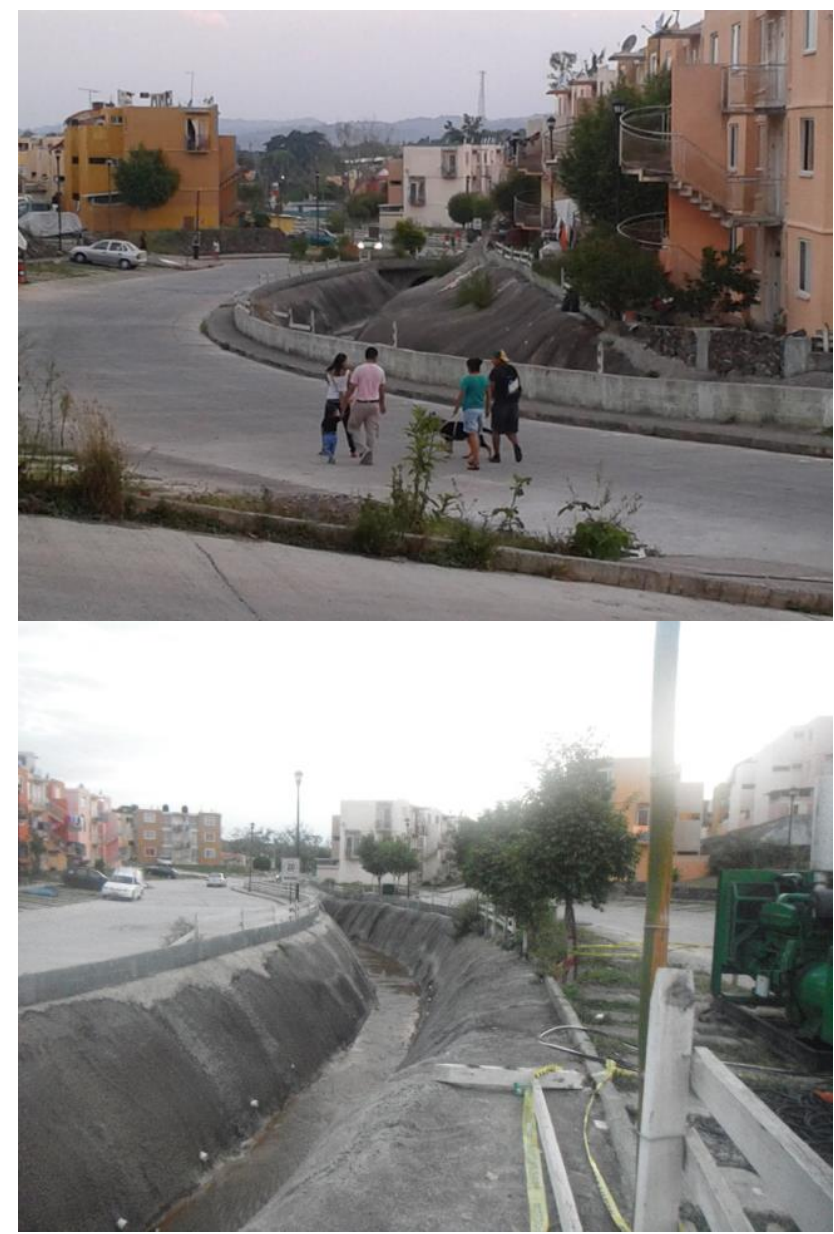

Figuras 6 y 7. Vía colectora, y canal de agua pluvial. Fuente: Elaboración propia.
Por otra parte, adjunta a la vía colectora se encuentra el canal de agua pluvial, que en época de lluvia tanto su capacidad como la del drenaje es insuficiente, y el agua sale del cauce (Figuras 6 y 7). Esto provoca la inundación de la vía colectora con agua sucia. También, se ha convertido en basurero: "hay gente cochina... por no dar dos tres pesos al de la basura decide tirar la basura en la calle" (ENT5-GR, 2014), situación a la cual se suma la falta de constancia del servicio de recolección de basura (una o dos veces a la semana). Con el tiempo, la basura, junto con las aguas negras, expele olor desagradable, es fuente de infección y pone en peligro la integridad física de los habitantes. Por otro lado, la existencia de pocos espacios públicos recreativos, donde los habitantes puedan realizar actividades como jugar, caminar o simplemente descansar, ha llevado a utilizar la vía colectora para tal fin. Dicha circunstancia alerta a los vecinos sobre todo por los niños, quienes no miden el peligro ante la ansiedad del juego: “... tratamos de estar al pendiente de los niños, más que nada por el canal... en cualquier momento se te pueden ir" (ENT2-GR, 2013).

En Los Mangos 1 y 2, la percepción de inseguridad incrementa en la vía local adjunta al espacio recreativo $A$ (Figuras 8 y 9). Las fachadas traseras de las viviendas de Los Mangos 2 y el borde cerrado del espacio recreativo $A$ confinan esta vía, lo que reduce la permeabilidad física y visual. Además, solo tiene banqueta en una de sus aceras, con un ancho de $0.80 \mathrm{~m}$ y con matorral, que apenas alcanza para la circulación de una persona. En cuanto al alumbrado público, hay lámparas, pero estas no funcionan. Así, ante la vulnerabilidad económica y social, dichas condiciones son propicias para la delincuencia, pues varias personas cuyo paso es obligado han sido víctimas.

Asimismo, en Los Mangos 2 se encuentra un canal de agua fluvial, el cual no cuenta con protección alguna en el borde (Figura 9), además está contaminado y expele mal olor; en temporada de lluvia se desborda e inunda las vías locales y viviendas contiguas, contribuyendo a la percepción de miedo y peligro.

En el espacio $C$ que está adjunto al canal de agua fluvial, también se percibe inseguridad (Figura 9). Es lugar de paso frecuente de los pobladores de la colonia popular contigua (Santa Fe). Los habitantes de Los Mangos 2 afirman que tales personas son quienes cometen delitos en el conjunto habitacional. A principios de 2014, en esta zona próxima al espacio $C$, secuestraron a un niño. Después del hecho, los vecinos decidieron poner elementos de protección en sus viviendas: rejas impermeables física y visualmente. Además, aumentó la atención hacia la presencia de personas extrañas. 


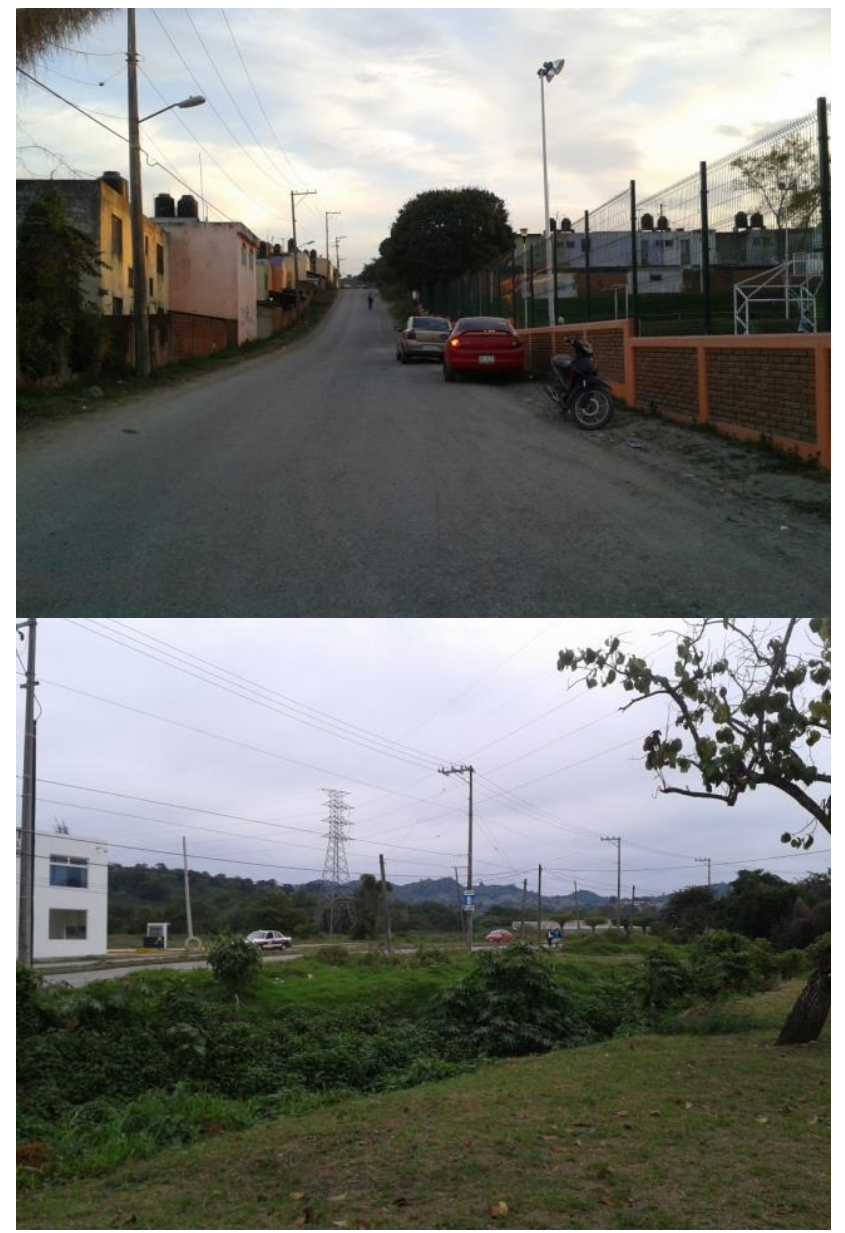

Figuras 8 y 9. Los Mangos 2, vía local, y canal de agua fluvial y espacio C. Fuente: Elaboración propia.

\section{Dimensión arquitectónica: diseño y calidad de construcción}

En Geovillas del Real, a lo largo de la vía colectora, la cerca de protección entre el canal de agua pluvial y aguas negras y la banqueta es de madera y se está pudriendo (Figura 10). Por lo anterior, los vecinos argumentan que varias personas se han accidentado, han caído o han estado a punto de hacerlo, sobre todo en temporada de Iluvia. Asimismo, el pavimento de esta vía se ha quebrado por la mala calidad del material, y la banqueta es insuficiente para acoger al peatón, pues está fracturada, obstruida (por postes, desniveles y socavones) y tiene entre 0.50 y $0.80 \mathrm{~m}$ de ancho.

En Los Mangos 1 y 2, la vía secundaria se curva de manera pronunciada a la altura del acceso principal del espacio público recreativo A (Figura 11), donde han ocurrido varios accidentes (los vehículos han impactado en ese punto).

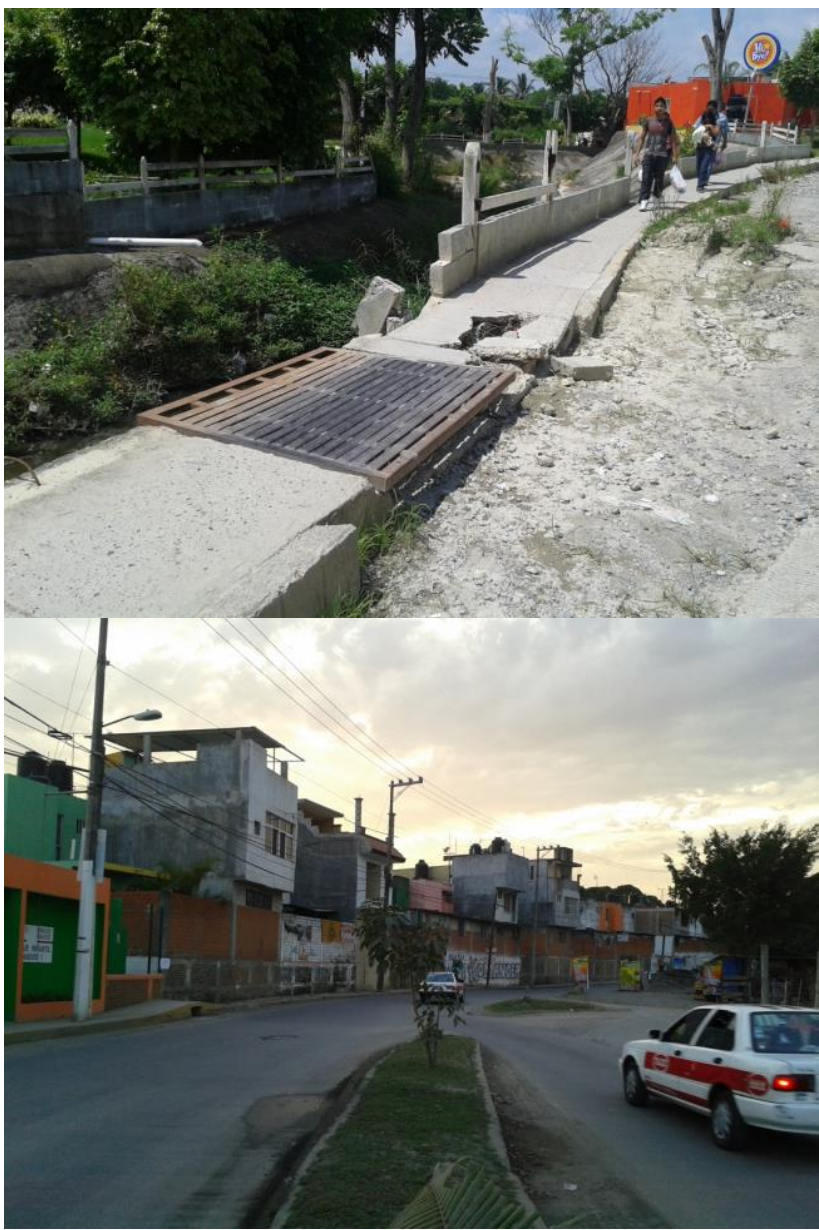

Figuras 10 y 11. Geovillas del Real (protección de madera), Los Mangos 1 y 2 (acceso principal de espacio $A$ ).

Fuente: Elaboración propia.

Como suele ocurrir, en el diseño no se toma en cuenta la opinión de los futuros usuarios ${ }^{6}$. Sin embargo, los residentes de Los Mangos 1 y 2 sí se enteraron con antelación acerca de la reubicación del acceso principal del espacio recreativo $A$, lo cual no significó que estuvieran de acuerdo con la obra. Primero se organizaron en juntas vecinales; después resolvieron, mediante oficio, que no había común acuerdo con la reubicación del acceso principal, ya que el sitio propuesto por el ayuntamiento era un punto de accidentes frecuentes, tal como lo expresa uno de los habitantes, quien insiste en lo invisible que son los habitantes para las autoridades: "La entrada, no se nos tomó nadie en cuenta... esa entrada... está muy peligrosa" (E4-LM1, 2015).

\footnotetext{
6 Por ejemplo, no se tomó en cuenta a los habitantes para la reconstrucción de 2014, realizada dentro del Programa de Mejoramiento
} 
El contexto urbano y arquitectónico expuesto contribuye a percibir peligro en el espacio recreativo $A$, que se conjuga con la presencia de grupos delictivos que aprovechan tal situación de vulnerabilidad.

\section{Dimensión social: incivilidad, vigilancia y desconfianza}

En Geovillas del Real, las zonas VI y VII son referentes de peligro, miedo e inseguridad. Están localizadas en la parte alta del terreno, son las más alejadas del acceso principal $(850 \mathrm{~m}$ ) y terminan en calle sin salida (Figuras 12 y 13$)$.
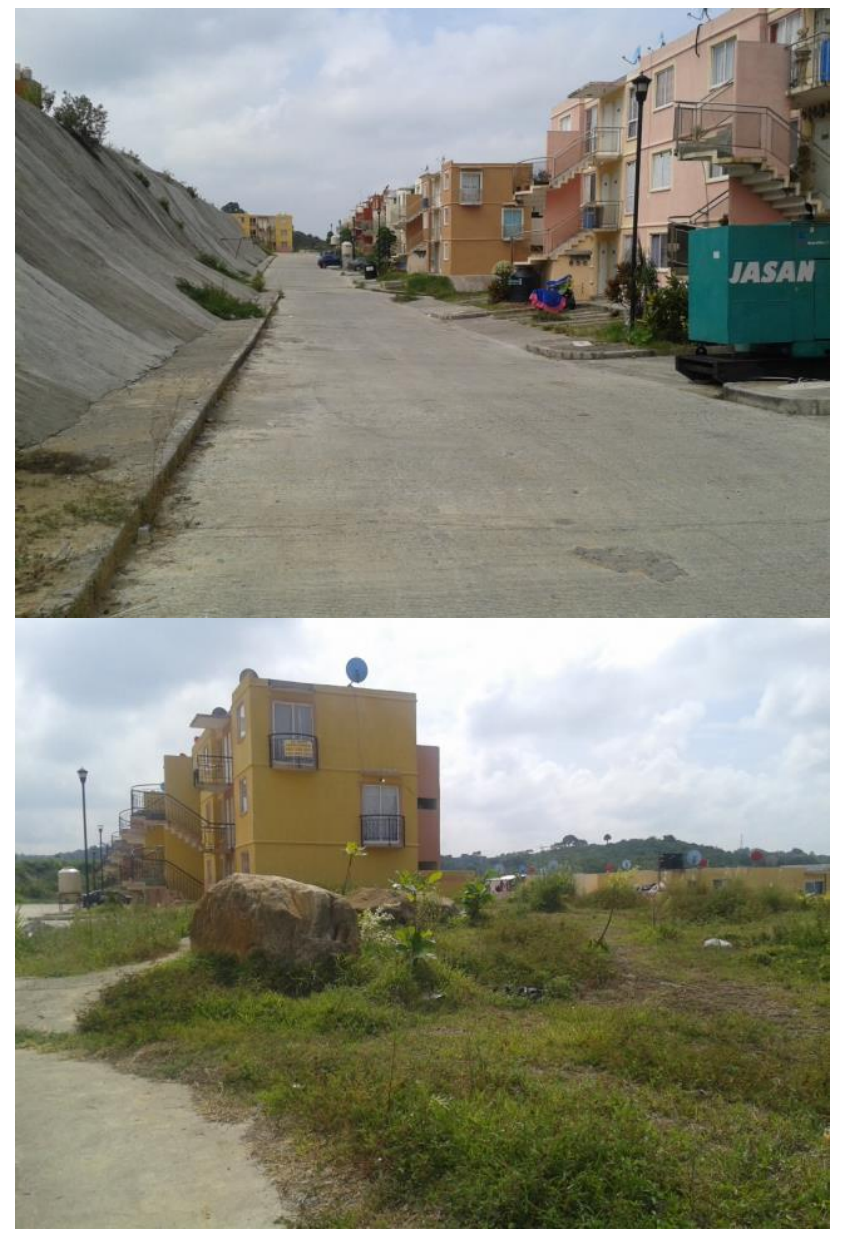

Figuras 12 y 13 . Zona VI y zona VII.

Fuente: Elaboración propia.

La imagen que se tiene de esta sección se vincula con la delincuencia, un habitante de la zona $\mathrm{V}$, por ejemplo, expone: "allá viven los malos, por eso ni me acerco, nadie se acerca" (ENC13-GR, 2015). Cabe decir que la mayoría de los habitantes asegura que es el lugar donde viven miembros del crimen organizado (los Zetas), por lo tanto, el robo y la invasión a viviendas deshabitadas es frecuente.
No obstante, se entrevistó a un habitante de la zona VI, quien dijo: "Aquí no pasa nada, es tranquilo, los malos viven más arriba, en la zona VIP, son vecinos y no se meten con uno" (ENC19-GR, 2015). Probablemente, en este comentario se evade la realidad inhóspita y se realza la supuesta tranquilidad, que en sí se la relaciona con la afluencia reducida de transeúntes ( 2 habitantes/hora), o como Lunecke (2016) sostiene, la violencia se ha normalizado.

Cabe mencionar otro factor que impacta directamente en la percepción de la seguridad: la suspensión del servicio de vigilancia en el conjunto habitacional que ha sido aprovechado para cometer varios hechos delictivos y robos, tal como los vecinos expresan:

... recién cuando se habían ido los guardias, ya no había seguridad, todo mundo quería hacer lo que quisiera y todo... y salió en el periódico que los mismos que nos hacían la limpieza, que nosotros les pagábamos, eran los rateros, porque ya nos tenían checaditos quiénes vivíamos, quiénes íbamos a trabajar (ENT1-GR, 2013).

Así pues, las personas externas al conjunto habitacional afectadas por el desempleo y el bajo salario se han valido de la situación, es decir, se han aprovechado de la vulnerabilidad de los residentes de Geovillas del Real, quienes tienen necesidad de ausentarse durante el día, o al menos medio día:

... aquí han robado bastante, hay gente que llega después de las once de la noche, del trabajo, las han jaloneado... y pues ahora si no contamos con una seguridad plena como antes, que estaban las 24 horas los vigilantes. Ahorita si se da cuenta, entran y salen cualquier persona, por eso ahorita estamos al cuidado de calle por calle... allá atrás hay espacio para que se pasen, se pasaban (ENT2-GR, 2014).

La falta de vigilancia y el aumento de los hechos delictivos son factores directamente relacionados. Como Cortés (2010, p.7) dice: “Contra estos 'otros', que representan la fragilidad y la precariedad de la existencia cotidiana, todas las sociedades se han dotado de múltiples argucias e instrumentos defensivos que permitieran preservar y mantener lo adquirido". Esto es, se establece una dualidad entre los residentes del conjunto habitacional y los externos, los otros, cuyo significado se vincula co $^{-}$la incertidumbre (Becerril, Méndez y Garrocho, 2013), existencia que provoca división, distancia, separación e inseguridad. Así, en septiembre de 2014, los habitantes decidieron formar un grupo de 
vigilancia vecinal que se encargara de controlar el acceso, a fin de salvaguardar los bienes, como medio defensivo ${ }^{7}$.

Sin embargo, Cortés (2010, p. 8) asegura que "a cada medida de seguridad que se toma aumenta la sensación de peligro en la que se vive, ninguna medida es suficiente, ningún acto bastante seguro; bien al contrario, los temores se perpetúan, aumentan y adquieren cada vez mayor protagonismo". Esto es factible porque la localización aislada del conjunto, su diseño urbano y arquitectónico, la falta de servicios básicos (iluminación, mantenimiento y vigilancia) y la crisis laboral que afecta a la zona metropolitana de Poza Rica y Coatzintla no ayudan a contrarrestar la delincuencia que ha plagado no solo a la zona norte de Veracruz, sino a todo el país. Además, a pesar de las medidas de seguridad, la delincuencia sigue operando, pues es el resultado del deterioro social (Velásquez, 2010), por lo que no se ha mitigado la sensación de vulnerabilidad de manera importante. De hecho, cuando se preguntó: ¿Cómo considera la incidencia de peleas, robos y delitos en el conjunto? las respuestas fueron las siguientes: $33,3 \%$ señaló que es alta; $56,7 \%$, regular y $10 \%$, baja.

Asimismo, cuando se cuestionó acerca del servicio de vigilancia: $36,7 \%$ lo calificó como bueno; $50 \%$, regular y $13,3 \%$, malo. No obstante, $50 \%$ opinó que es peor en comparación con el barrio anterior donde vivía, 30\% indicó que es mejor y $20 \%$ dijo que es igual. Ante la presencia de personas externas: $86,7 \%$ expresó sentir desconfianza y $13,3 \%$ mencionó no sentirla. Más aún, la desconfianza aumenta cuando algún miembro de la familia usa el espacio público: $60 \%$ percibía desconfianza, pero no así el restante $40 \%$.

En Los Mangos 1 y 2, el acceso principal es referente de inseguridad: en el borde derecho colinda con un terreno baldío y en el izquierdo con la fachada delantera cerrada de algunas viviendas (Figura 14). Por tal razón, la permeabilidad visual entre el espacio público y privado es limitada. A esto se suma la falta de alumbrado público, que lo convierte en un lugar propicio para los delincuentes. Al respecto, un vecino expresa:

... asaltaron a mi hija y a otras gentes, ese lugar es inseguro sin luz, no sé por qué pusieron la imagen de la Lupita, si ella no nos cuida, yo decía que mejor una caseta de vigilancia, pero ya ve como es la gente. (ENC4-LM1, 2015).

Ahora bien, el nicho religioso en honor a la virgen de Guadalupe se ha convertido en símbolo de protección para algunos, pero solo ha servido para que las personas no tiren basura en el área (Figura 15).

Una jardinera en banqueta provoca mixtura de sensaciones, según la hora del día (Figura 16). La banqueta tiene solo $0.60 \mathrm{~m}$ de ancho y tiene una jardinera con enredaderas y arbustos. Durante el día es transitada con frecuencia; es agradable porque la sombra protege del intenso calor. No obstante, durante la noche es referente de miedo, debido a la falta de iluminación, pues las lámparas públicas no funcionan; la falta de permeabilidad visual entre el espacio privado y público es limitada, porque las plantas obstruyen la visibilidad. Características que son aprovechadas por la delincuencia, pues se han presentado asaltos a transeúntes.

Cuando se preguntó: ¿Cómo considera la incidencia de peleas, robos y delitos en el conjunto? Las respuestas fueron las siguientes: para $36,7 \%$ la repercusión es alta, $36,7 \%$ manifestó que es media y solo $26,7 \%$ dijo que es baja. En relación con el servicio de vigilancia, 76,7\% lo calificó como malo, $23,3 \%$ como regular y solo $10 \%$ como bueno; $80 \%$ opinó que es peor en comparación con el barrio anterior, $10 \%$ indicó que es mejor y $10 \%$ dijo que es igual. Además, la población manifestó tener desconfianza cuando algún miembro de la familia usa el espacio público: $56,7 \%$ percibe falta de confianza, mientras que $43,3 \%$ no la percibe. Se pone énfasis en la percepción de peligro ante la presencia de personas externas: 86,6\% expresó que no confía y $13,3 \%$ dijo que no experimentaba esa sensación. Persiste el miedo al otro, al que no es igual; probablemente esto se debe a los hechos delictivos ocurridos en los últimos años a consecuencia de la crisis laboral y social que padece la región.

\footnotetext{
7 Para remunerar a los vigilantes se acordó una cuota de 25 pesos semanales por vivienda, aunque es importante mencionar que solo $10 \%$ de los habitantes la paga.
} 


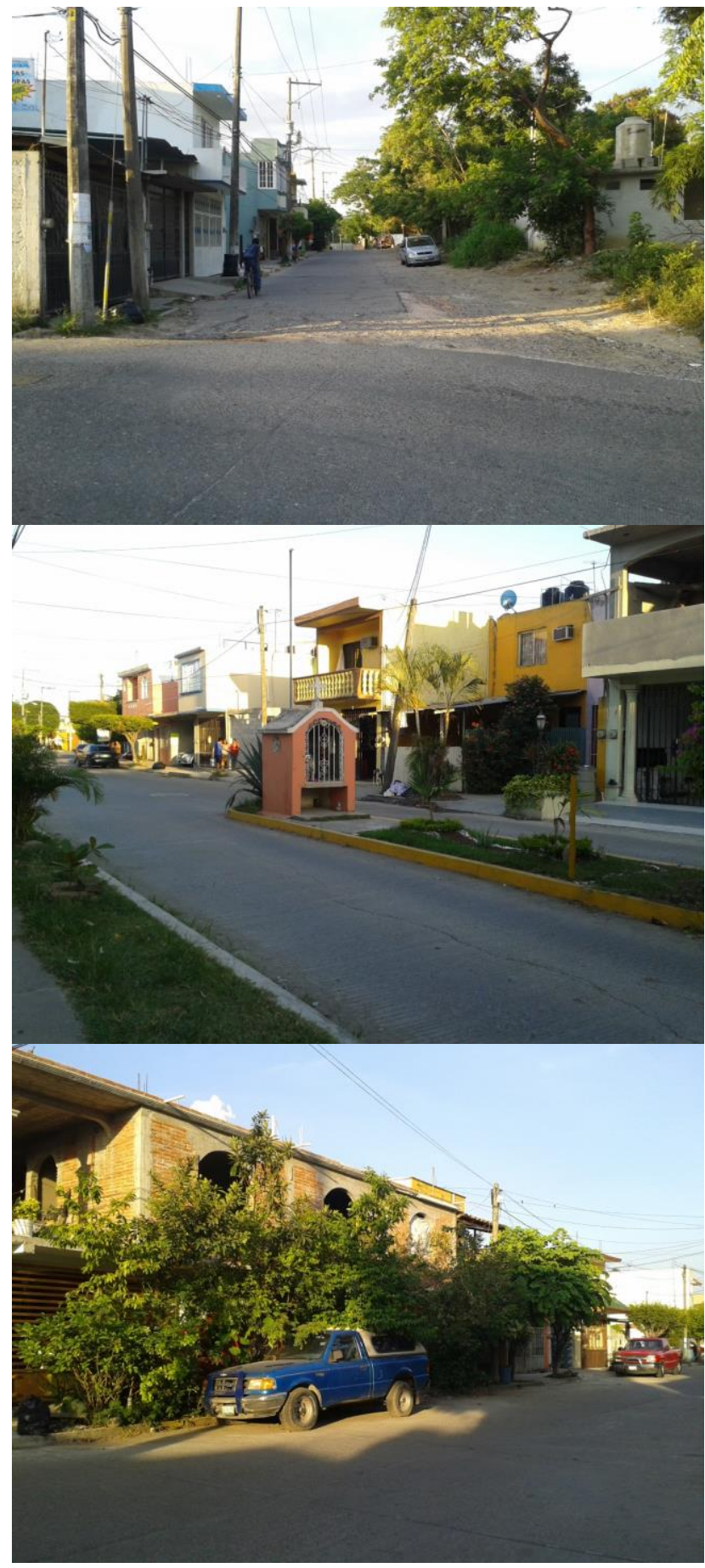

Figuras 14, 15 y 16. Los Mangos 1: acceso principal, nicho religioso (G), y jardinera en banqueta (a).

Fuente: Elaboración propia.

\section{Conclusiones}

En cuanto al objeto de estudio que compete a este análisis, en materia de diseño urbano y arquitectónico se ha perdido el sentido social del espacio público, lo cual ha debilitado la percepción de seguridad en sus usuarios. Esto se debe a factores y prácticas que restringen $o$ transforman la apropiación y uso cotidiano del espacio público, lo cual ha tenido consecuencias en la calidad de vida, como a continuación se exponen.

El amurallado de los conjuntos impide el intercambio físico y visual entre espacio público y privado; es frecuente limitar la accesibilidad física y visual por los muros ciegos de las fachadas traseras y laterales de las viviendas. La configuración semicerrada de las tres escalas afecta la percepción de seguridad.

Además, la localización, las características del terreno y el diseño arquitectónico mal resuelto traen consigo referentes de peligro que ponen en riesgo la vida de los habitantes de estos enclaves. A lo que se suma la mala calidad del material de construcción $y$, por ende, su deterioro prematuro, y la carencia de servicios básicos como mantenimiento, iluminación y vigilancia.

El problema de mantenimiento se debe a la falta de compromiso y organización de algunos habitantes, debido a la inversión de tiempo que conlleva, o bien, por la simple indiferencia; esto se agrava con la limitada atención del municipio; la incapacidad económica o falta de voluntad. Aún más, por la falta de solvencia económica de los propios habitantes que suscita una cultura de no pago de las cuotas colectivas. La iluminación o alumbrado público es otro factor que condiciona la apropiación y el uso del espacio público. A esto se suma la falta de vigilancia, cuya carencia implica que los habitantes se sientan vulnerables ante personas ajenas al conjunto, a los otros.

En particular, la crisis económica de la industria petrolera ha tenido como resultado la falta de empleo, por tanto, el aumento de la delincuencia local, a lo que se suma el incremento de la presencia del crimen organizado. Así, el prejuicio de uso y la presencia de personas externas al conjunto con frecuencia se relaciona con la desconfianza y el miedo a ser violentados, lo cual afecta la obtención de satisfacción. Con lo anterior, se deduce que el espacio público no se construye solo o simplemente por delimitarlo físicamente, sino que obedece a un conjunto de leyes sociales que generan o no la apropiación, y el sentido de apropiación está relacionado con el sentido de confortabilidad y/o satisfacción.

En general, a partir de la implementación de la política habitacional mercantil, que ha sustentado al sistema industrial en la construcción de conjuntos de vivienda de interés social, los menos beneficiados son los habitantes de dichos enclaves; el capital privado ha tomado las riendas del financiamiento, el diseño y la construcción, mientras que el papel del Estado se ha ceñido a la gestión. En este sentido, se propone que se haga visible la participación social equitativa en el diseño y construcción de los conjuntos habitacionales de interés social: que 
considere tanto a los habitantes o futuros beneficiarios de las viviendas como al capital privado y al Estado[B]

\section{Referencias}

Alguacil, J. (2000). Calidad de vida y modelo de ciudad. Boletín CF+S. Biblioteca CF+S. Ciudades para un Futuro más Sostenible. Madrid: Instituto Juan de Herrera. Recuperado de http://habitat.aq.upm.es/boletin/n15 /ajalg.html

Becerril, T., Méndez, J., y Garrocho C. (2013). Urbanizaciones cerradas y transformaciones socioespaciales en Metepec, Estado de México. Revista EURE, 39(117), 191-213. http://dx.doi. org/10.4067/S0250-71612013000200009

Bentley, I., Alcock, A., Murrain, P., MacGlynn, S., y Smith, G. (1999). Entornos vitales. Hacia un diseño urbano y arquitectónico más humano. Manual práctico. Barcelona: Gustavo Gili.

Carmona, M., Health, T., Oc, T., \& Tiesdell, S. (2003). Public places urban spaces the dimensions of urban design. Oxford: Architectural Press.

Cortés, J. (2010). La ciudad cautiva. Control y vigilancia en el espacio urbano. Madrid: Akal.

Cruz Muñoz, F., e Isunza, G. (2017). Construcción del hábitat en la periferia de la Ciudad de México. Estudio de caso en Zumpango. Revista EURE, 43(129), 187-207. http://dx.doi.org/10.4067/S025071612017000200009.

Durán, A., Esquivel, M. T., y Giglia, A. (2000). Condiciones de vida y vivienda de interés social en la Ciudad de México. México: UAM-Azcapotzalco.

Fraile, P. (2007). La percepción de seguridad: entre el delito, el conflicto y la organización del espacio. SCRIPTA NOVA. Revista Electrónica de Geografía y Ciencias Sociales, 62(249). Recuperado de: http:// www.ub.es/geocrit/sn/sn-24562.htm

García, F. A. y Peralta, M. P. (2016). Las urbanizaciones multifamiliares cerradas y su entorno urbano: una nueva geografía simbólica en la ciudad de Cali (Colombia). Revista EURE, 42(126), 77-96. http://dx. doi.org/10.4067/S0250-71612016000200004

Giglia, A. (2012). El habitar y la cultura. Perspectivas teóricas y de investigación. México: UAM-Iztapala.

González, R. (17 de enero de 2014). En el abandono, cinco millones de casas hipotecadas. La Jornada (México), p. 22.

Holahan, C. (2011). Psicología ambiental. México: Limusa.
Jacobs, J. (2011). Muerte y vida de las grandes ciudades. Madrid: Capitán Swing.

Jacobs, J. (1973). Muerte y vida de las grandes ciudades. Madrid: Ediciones Península.

Jasso, C. L. (2013). Percepción de inseguridad en México. Revista Mexicana de Opinión Pública, 15, 12-29. http://dx.doi.org/10.1016/S1870-7300(13)72319-6

Jiménez, J. E. y Garnica, R. (2016). Metodología para la medición del déficit cualitativo de espacio público en Colombia: un indicador clave del ordenamiento territorial. Revista de Urbanismo, 35, 69-99. http://d x.doi.org/10.5354/0717-5051.2016.42481

Lunecke, A. (2016). Inseguridad ciudadana y diferenciación social en el nivel microbarrial: el caso del sector Santo Tomás, Santiago de Chile. Revista EURE, 42(125), 109-129. http://dx.doi.org/10.4067 /SO250-71612016000100005

Maerker, D. (25 de septiembre de 2015). Cruda realidad: Poza Rica sin empleos, sin inversión y sin desarrollo. Punto de Partida. https://www.youtube.com/watch ?v=JMWLvWhOMjA

Mejía, M. (2012). Habitabilidad en la vivienda social en edificios para población reasentada. El caso de Medellín, Colombia. Revista EURE, 38(114), 203-227. http://dx.doi.org/10.4067/S0250-7161201200020000 $\underline{8}$

Maycotte, E. (2010). Espacios abiertos y calidad de vida en conjuntos habitacionales organizados en condominio. México: Infonavit y la Universidad Autónoma del Estado de México

Nava, M. (2012). Las fuerzas armadas y el crimen organizado en la democracia mexicana. Revista de EI Colegio de San Luis, 2(3), 324-341. http://www.reda lyc.org/articulo.oa?id=426239575015

Nussbaum, M. y Sen, A. (1996). La calidad de vida. México: Fondo de Cultura Económica.

Programa Nacional de Vivienda 2014-2018. Diario Oficial de la Federación, 30 de abril de 2014. Recuperado de http://dof.gob.mx/nota detalle.php?codigo $=5342865 \&$ fecha $=30 / 04 / 2014$

Programa Municipal de Desarrollo Urbano de Poza Rica (2009). México: Gobierno del Estado de Veracruz de Ignacio de la Llave, Xalapa-Enríquez, Veracruz. 
Rabotnikof, N. (2011). En busca de un lugar común. El espacio público en la teoría pública contemporánea. México: Universidad Nacional Autónoma de MéxicoInstituto de Investigaciones Filosóficas.

Rueda, S. (1997). Habitabilidad y calidad de vida. Biblioteca $\mathrm{CF}+\mathrm{S}$ Ciudades para un futuro más sostenible. Disponible en http://habitat.aq.upm. es/cs/p2/a005.html.

Schteingart, M. y Patiño, L. (2006). El marco legislativo, programático e institucional de los programas habitacionales. En R. Coulomb y M. Schteingart (Coords.), Entre el Estado y el mercado. La vivienda en el México de hoy (pp. 153-192). México: UAMAzcapotzalco y Miguel Ángel Porrúa.

Segovia, O. (2005). Habitar en conjuntos de vivienda social: ¿cómo construir identidad, confianza y participación? En Rodríguez, A. y Sugraynes, A. (Eds.), Los con Techo, un desafío para la política de vivienda social (pp. 287-318) Santiago de Chile: SUR Corporación de Estudios Sociales.

Urbano Paz, L. y Delpino Chamy, M. (2015). Convivencialidad y seguridad en espacios comunitarios del movimiento moderno. Estudio de la Remodelación Paicaví, Concepción. Revista de Urbanismo, 33, 37-51. http://dx.doi.org/10.5354/0717-5051.2015.36649

Velásquez, C. A. (2010). Crimen organizado: orden divergente y vecindarios urbanos vulnerables. Revista EURE, 36(108), 49-74. http://dx.doi.org/10.406 7/S0250-71612010000200003

World Health Organization, WHO. (2018). WHOQOL: Measuring Quality of Life. Recuperado de http://www.who.int/healthinfo/survey/whogolqualityoflife/en/ 